\title{
Heart Failure with Preserved Ejection Fraction in a Postpartum Patient with Superimposed Preeclampsia and COVID-19
}

\author{
Rachel G. Sinkey, MD ${ }^{1,2}$ Indranee Rajapreyar, $\mathrm{MD}^{3}$ \\ Jodie Dionne-Odom, $\mathrm{MD}^{4}$ Steven M. Pogwizd, $\mathrm{MD}^{3}$ \\ ${ }^{1}$ Division of Maternal-Fetal Medicine, Department of Obstetrics and \\ Gynecology, University of Alabama at Birmingham, Birmingham, \\ Alabama \\ ${ }^{2}$ Center for Women's Reproductive Health, University of Alabama at \\ Birmingham, Birmingham, Alabama \\ ${ }^{3}$ Division of Cardiovascular Disease, Department of Medicine, \\ University of Alabama at Birmingham, Birmingham, Alabama \\ ${ }^{4}$ Division of Infectious Disease, Department of Medicine, University of \\ Alabama at Birmingham, Birmingham, Alabama
}

Lindsay S. Robbins, MD ${ }^{1,2}$

Brian M. Casey, MD ${ }^{1,2}$ Alan T.N. Tita, MD, $\mathrm{PhD}^{1,2}$

Address for correspondence Rachel Sinkey, MD, 1700 6th Avenue, South, 176 F, Suite 10270, Birmingham, AL 35249

(e-mail: Rsinkey@uabmc.edu).

Am J Perinatol Rep 2020;10:e165-e168.

\begin{abstract}
Keywords

- COVID-19

- heart failure with preserved ejection fraction

- hypertensive emergency

- preeclampsia

Our understanding of COVID-19 in pregnant and postpartum women is rapidly evolving. We present a case from March 2020 of a 25-year-old G2P2002 whose delivery was complicated by preeclampsia with severe features who presented to the emergency department 9 days after cesarean delivery with chest tightness and dyspnea on exertion. On presentation she had severe hypertension, pulmonary edema, elevated brain natriuretic peptide, and high-sensitivity troponin-l, suggesting a diagnosis of hypertensive emergency leading to heart failure with a preserved ejection fraction resulting in pulmonary edema and abnormal cardiac screening tests. However, bilateral opacities were seen on a computed tomography of the chest, and COVID-19 testing was positive. A high index of suspicion for both COVID-19 and cardiovascular complications are critical for optimal patient outcomes and protection of health care workers.
\end{abstract}

Severe acute respiratory syndrome-coronavirus-2 (SARSCoV-2), the virus that leads to COVID-19, was first reported in Wuhan, China, in December 2019. ${ }^{1}$ The high-infectivity rate of this virus in an era of widespread global travel led to an unprecedented international pandemic infecting over two million individuals and claiming nearly 200,000 lives at the time of submission. ${ }^{2}$ Rapid spread of a never-beforeseen disease leaves a unique absence of clinical guidance. Data on COVID-19 in pregnant women and their neonates are sparse. Emerging data in nonpregnant adults suggests COVID-19 is associated with cardiac injury in 7 to $17 \%$ of hospitalized COVID-19 patients, ${ }^{3}$ but cardiovascular sequa- lae in pregnancy are unknown. We report a case of a postpartum patient who presented early in the COVID-19 pandemic highlighting the importance of maintaining a broad differential diagnosis and widespread testing for COVID-19.

\section{Case}

A 25-year-old African American G2P1001 woman whose pregnancy was complicated by chronic hypertension on labetalol 600-mg twice daily, class-III obesity with a body mass index of $68 \mathrm{~kg} / \mathrm{m}^{2}$ and iron-deficiency anemia presented for a received

April 20, 2020

accepted after revision

April 30, 2020
DOI https://doi.org/

10.1055/s-0040-1712926. ISSN 2157-6998.
Copyright $\odot 2020$ by Thieme Medical Publishers, Inc., 333 Seventh Avenue, New York, NY 10001, USA. Tel: +1(212) 760-0888.
License terms

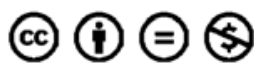


repeat cesarean delivery (CD) at $39^{0 / 7}$ weeks. On admission, she was asymptomatic, but she was noted to have severe hypertension $(172 / 104 \mathrm{~mm} \mathrm{Hg})$ and required intravenous antihypertensives. Laboratory evaluation revealed normal renal (creatinine $0.5 \mathrm{mg} / \mathrm{dL}$ ), liver (aspartate transaminase 36 units/L), and hematopoietic (white blood cell count $10 \times 10^{3} / \mathrm{cmm}$, total lymphocyte count $1,844 / \mathrm{dL}$ (normal $1,000-4,800 / \mathrm{dL}$ ), and platelets $293 \times 10^{3} / \mathrm{cmm}$ ) function. A urine protein-to-creatinine ratio resulted at 7.3 , significantly increased from her baseline of 0.14 . The patient was diagnosed with superimposed preeclampsia with severe features based on blood pressure criteria. Magnesium sulfate was administered to reduce the risk of eclampsia, and an uncomplicated repeat $\mathrm{CD}$ and bilateral tubal ligation was performed. Magnesium sulfate was administered for 24 hours postpartum, and her labetalol was increased to $800-\mathrm{mg}$ twice daily due to persistently elevated blood pressures. The patient and her neonate daughter, weighing $4,170 \mathrm{~g}$, both did well in the postpartum period. She opted to formula feed her infant, and both were discharged to home on postoperative day3. The patient's last recorded vital signs prior to discharge included a heart rate of 92 beats per minute, respiratory rate of 20 breaths per minute, and blood pressure of $146 / 89 \mathrm{~mm} \mathrm{Hg}$.

On postoperative day 9, 6 days after hospital discharge, the patient presented to the emergency department with 3 days of chest tightness and 1 day of progressive shortness of breath and dyspnea on exertion with substernal, nonradiating chest pain. She denied cough, fevers or chills, and described severe dyspnea with minimal exertion and stated that "just moving in bed" caused shortness of breath. Her blood pressure reading was 188/126 mm Hg, heart rate 95 beats per minute, temperature $97.1^{\circ} \mathrm{F}$, respiratory rate 30 breaths per minute and peripheral oxygen saturation $92 \%$, which improved to $99 \%$ on 2 L of oxygen delivered via nasal canula. A chest X-ray demonstrated interstitial and airspace opacities, a pleural effusion and a mildly enlarged heart ( - Fig. 1). An electrocardiogram demonstrated normal sinus rhythm without acute ischemic changes. White blood cell count was $10.87 \times 10^{3} \mathrm{cmm}$, serum creatinine was

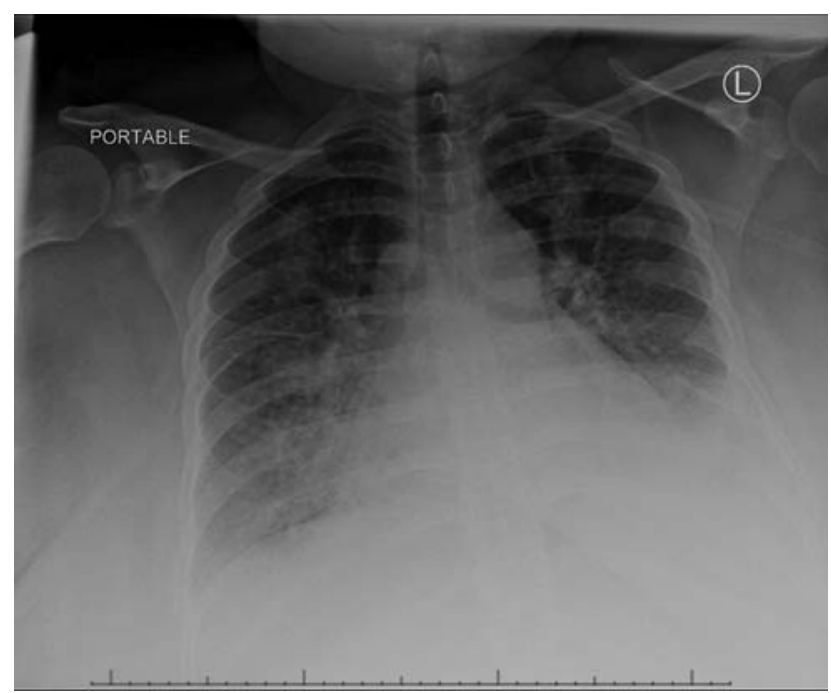

Fig. 1 Portable chest X-ray demonstrating interstitial and airspace opacities, pleural effusion and a mildly enlarged cardiac silhouette.
$0.6 \mathrm{mg} / \mathrm{dL}$, brain natriuretic peptide was $253 \mathrm{pg} / \mathrm{mL}$ (normal range: $0-100 \mathrm{pg} / \mathrm{mL}$ ), and high-sensitivity troponin-I at presentation and 2 and 7 hours later were 20,25, and $23 \mathrm{ng} / \mathrm{L}$ (normal range: $3-20 \mathrm{ng} / \mathrm{mL}$ ), respectively. The differential diagnosis at this time included but was not limited to hypertensive emergency leading to cardiac dysfunction, a non-ST-elevation myocardial infarction, pulmonary thromboembolism, and peripartum cardiomyopathy.

Then $20 \mathrm{mg}$ of intravenous (IV) labetalol, $40 \mathrm{mg}$ of IV furosemide, and $0.4 \mathrm{mg}$ of sublingual nitroglycerin were administered. The patient reported improved symptoms, her systolic blood pressure decreased to $<160 \mathrm{~mm} \mathrm{Hg}$, and the obstetrics service was consulted for admission. Given multiple risk factors for a pulmonary embolism, a computed tomography angiogram (CTA) of the chest was performed. The chest CTA showed no evidence of thromboembolism and moderate volume pleural effusion (right $>$ left) with compressive atelectasis. In addition, there was scattered air space consolidation in bilateral lung fields with associated bronchial wall thickening, suggestive of infectious etiology (-Fig. 2). Cardiology service was consulted due to chest pain and abnormal troponin results. They recommended continued diuresis with IV furosemide and blood pressure control with IV labetalol, as well as the request for a formal echo that demonstrated normal left ventricular (LV) systolic function, ejection fraction 55 to $60 \%$, normal LV wall thickness, no significant valvular abnormalities, and a ratio of early mitral inflow velocity estimated by the Doppler and mitral annular early diastolic velocity by tissue Doppler (E/e') of 15.4 suggestive of elevated left-sided filling pressures (normal $\mathrm{E} / \mathrm{e}^{\prime}<14$ ). A diagnosis of heart failure with preserved ejection fraction (HFpEF) was reached based on the patient's $\mathrm{H}_{2}$ FPEF score, clinical symptoms, and elevated brain natriuretic peptide (BNP). ${ }^{4}$ However, based on bilateral ground-glass opacities on chest CTA, COVID-19 testing was

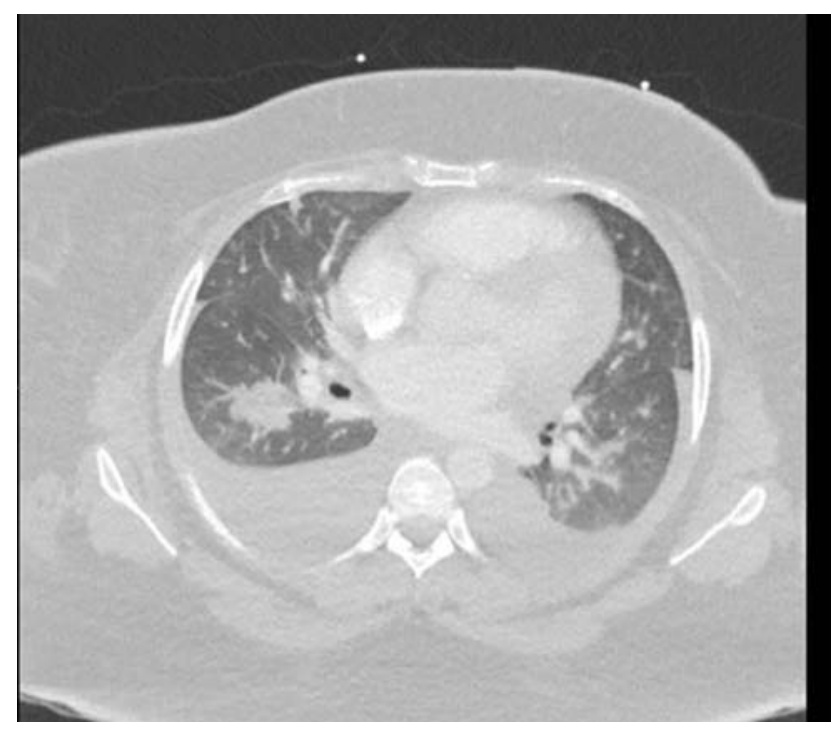

Fig. 2 Computed tomography angiogram of the chest depicting scattered air space consolidation in bilateral lung fields with associated bronchial wall thickening suggestive of an infectious etiology, moderate volume pleural effusions (right $>$ left), compressive atelectasis, and no evidence of pulmonary thromboembolism. 
recommended. A nasopharyngeal swab was collected, and COVID-19 testing was sent to a regional testing center. Given limited testing capabilities early in the pandemic, the expected turnaround time was 3 to 4 days. She was placed on respiratory precautions and treated as a person under investigation (PUI). By hospital day 3, her respiratory complaints resolved; she was euvolemic; her blood pressure was controlled with carvedilol $25 \mathrm{mg}$ twice per day, amlodipine 10-mg daily, and enalapril $2.5 \mathrm{mg}$ daily; and she was deemed stable for discharge home with self-isolation pending the results of her test. Given her continued COVID-19 PUI status, arrangements were made for her discharge medications to be picked up and delivered to her home, and her infant was able to be temporarily housed with the patient's family during her quarantine.

COVID-19 RNA polymerase chain reaction (PCR) test resulted positive hours after her hospital discharge, amounting to a 2-day turnaround time. The patient was notified of the result by phone and advised to remain in quarantine for 7 days. If fever or respiratory symptoms persisted on days 5 to 7 of the quarantine, she was instructed to remain in isolation for an additional 72 hours after symptom resolution per infectious diseases. The patient's mother cared for the newborn for the duration of the patient's self-isolation. At the time of submission, for 2 weeks postdischarge, the patient remained asymptomatic, and the patient's family members and infant are doing well without symptoms of COVID-19. Close echocardiographic follow-up is planned.

\section{Discussion}

Postpartum severe hypertension among women with chronic hypertension in our patient population is $13 \% .^{5}$ Initially, the singular diagnosis that appeared to explain this patient's constellation of symptoms, vitals, examination, and radiologic findings was hypertensive emergency leading to HFpEF which resulted in pulmonary edema, elevated BNP, and an elevated troponin. ${ }^{6,7}$ However, bilateral ground-glass opacities on chest CTA were consistent with bronchopneumonia reported in Chinese patients with COVID-19. ${ }^{8,9}$ Her HFpEF was likely exacerbated by the concomitant COVID-19 pneumonia, as patients with heart failure experience exacerbations with infectious processes. ${ }^{10}$ The bilateral pleural effusions in this patient are a unique finding not usually present in patients with COVID-19; of critically ill patients with COVID-19 from Seattle, no patients had coexisting pleural effusions. ${ }^{11}$

Myocardial injury with elevation in cardiac troponin has been reported in 20to 30\% of hospitalized COVID-19 patients. ${ }^{12}$ Myocardial injury could be a result of direct injury to the myocardium causing myocarditis, or indirect injury because of hypoxia due to acute respiratory distress syndrome or cytokine storm. Reversible diastolic dysfunction has been observed in patients with SARS-CoV. ${ }^{13}$ The hypoxia from COVID-19 broncopneumonia likely contributed to microvascular ischemia with resultant diastolic dysfunction and pulmonary edema and pleural effusions. Hypertension is a significant risk factor for complicated courses with COVID-19 infection; ${ }^{14}$ it is postulated that inflammatory and endothelial alterations may place patients at increased risk of complicated courses. Endothelial dysfunction from this patient's chronic hypertension, exacerbated by superimposed preeclampsia with severe features, could have been a contributing factor to her more complicated course.

The public health implications of a positive diagnosis after hospital discharge are significant. This patient presented early in the pandemic when testing was performed for patients with known exposures, travel from high-risk areas, and symptomatic patients with fever or cough. This patient had no known exposures, was afebrile and did not have a cough; at the time she did not meet testing criteria for COVID-19. Our institution has since implemented universal COVID-19 screening upon hospital admission. If diagnostic resources allow, we urge strong consideration of universal screening of hospitalized patients. Ideally, the turnaround time for results should be short.

\section{Conclusion}

COVID-19 infection is associated with cardiovascular complications. ${ }^{12}$ Providers should maintain a high index of suspicion and screen pregnant and postpartum patients with cardiovascular symptoms or shortness of breath with a chest $\mathrm{X}$-ray, electrocardiogram, troponin, and brain natriuretic peptide. Echocardiogram should be performed if screening tests are abnormal. Patients with preeclampsia have an increased risk of future cardiovascular disease; ${ }^{15}$ it is unknown if a diagnosis of HFpEF associated with COVID-19 compounds this future risk.

Conflict of Interest

None declared.

\section{References}

1 Zhu N, Zhang D, Wang W, et al; China Novel Coronavirus Investigating and Research Team. A novel coronavirus from patients with pneumonia in China, 2019. N Engl J Med 2020;382(08):727-733

2 Johns Hopkins University Coronavirus Resource Center. COVID-19 Dashboard by the Center for Systems Science and Engineering (CSSE) at Johns Hopkins University. Available at: https://coronavirus.jhu.edu/map.html. Accessed April 18, 2020

3 Driggin E, Madhavan MV, Bikdeli B, et al. Cardiovascular considerations for patients, health care workers, and health systems during the coronavirus disease 2019 (COVID-19) pandemic. J Am Coll Cardiol 2020;S0735-1097(20)34637-4 (e-pub ahead f print) . Doi: 10.1016/j.jacc.2020.03.031

4 Reddy YNV, Carter RE, Obokata M, Redfield MM, Borlaug BAA. A simple, evidence-based approach to help guide diagnosis of heart failure with preserved ejection fraction. Circulation 2018;138 (09):861-870

5 Glover AV, Tita A, Biggio JR, Anderson SB, Harper LM. Incidence and risk factors for postpartum severe hypertension in women with underlying chronic hypertension. Am J Perinatol 2019;36 (07):737-741

6 Whelton PK, Carey RM, Aronow WS, et al. 2017 ACC/AHA/AAPA/ ABC/ACPM/AGS/APhA/ASH/ASPC/NMA/PCNA guideline for the prevention, detection, evaluation, and management of high blood pressure in adults: a report of the American College of Cardiology/ American Heart Association Task Force on clinical practice guidelines. Hypertension 2018;71(06):1269-1324 
7 Oktay AA, Shah SJ. Diagnosis and management of heart failure with preserved ejection fraction: 10 key lessons. Curr Cardiol Rev 2015;11(01):42-52

8 Zhang S, Li H, Huang S, You W, Sun H. High-resolution CT features of 17 cases of corona virus disease 2019 in Sichuan province, China. Eur Respir J 2020 (e-pub ahead of print) . Doi: 10.1183/13993003. 00334-2020

9 Shi H, Han X, Jiang N, et al. Radiological findings from 81 patients with COVID-19 pneumonia in Wuhan, China: a descriptive study. Lancet Infect Dis 2020;20(04):425-434

10 Ather S, Chan W, Bozkurt B, et al. Impact of noncardiac comorbidities on morbidity and mortality in a predominantly male population with heart failure and preserved versus reduced ejection fraction. J Am Coll Cardiol 2012;59(11):998-1005
11 Bhatraju PK, Ghassemieh BJ, Nichols M, et al. Covid-19 in critically ill patients in the Seattle region - case series. N Engl J Med 2020

12 Madjid M, Safavi-Naeini P, Solomon SD, Vardeny O. Potential effects of coronaviruses on the cardiovascular system: a review. JAMA Cardiol 2020

13 Li SS, Cheng CW, Fu CL, et al. Left ventricular performance in patients with severe acute respiratory syndrome: a 30-day echocardiographic follow-up study. Circulation 2003;108(15):1798-1803

14 Zhou F, Yu T, Du R, et al. Clinical course and risk factors for mortality of adult inpatients with COVID-19 in Wuhan, China: a retrospective cohort study. Lancet 2020;395(10229):1054-1062

15 Smith GN, Louis JM, Saade GR. Pregnancy and the postpartum period as an opportunity for cardiovascular risk identification and management. Obstet Gynecol 2019;134(04):851-862 